
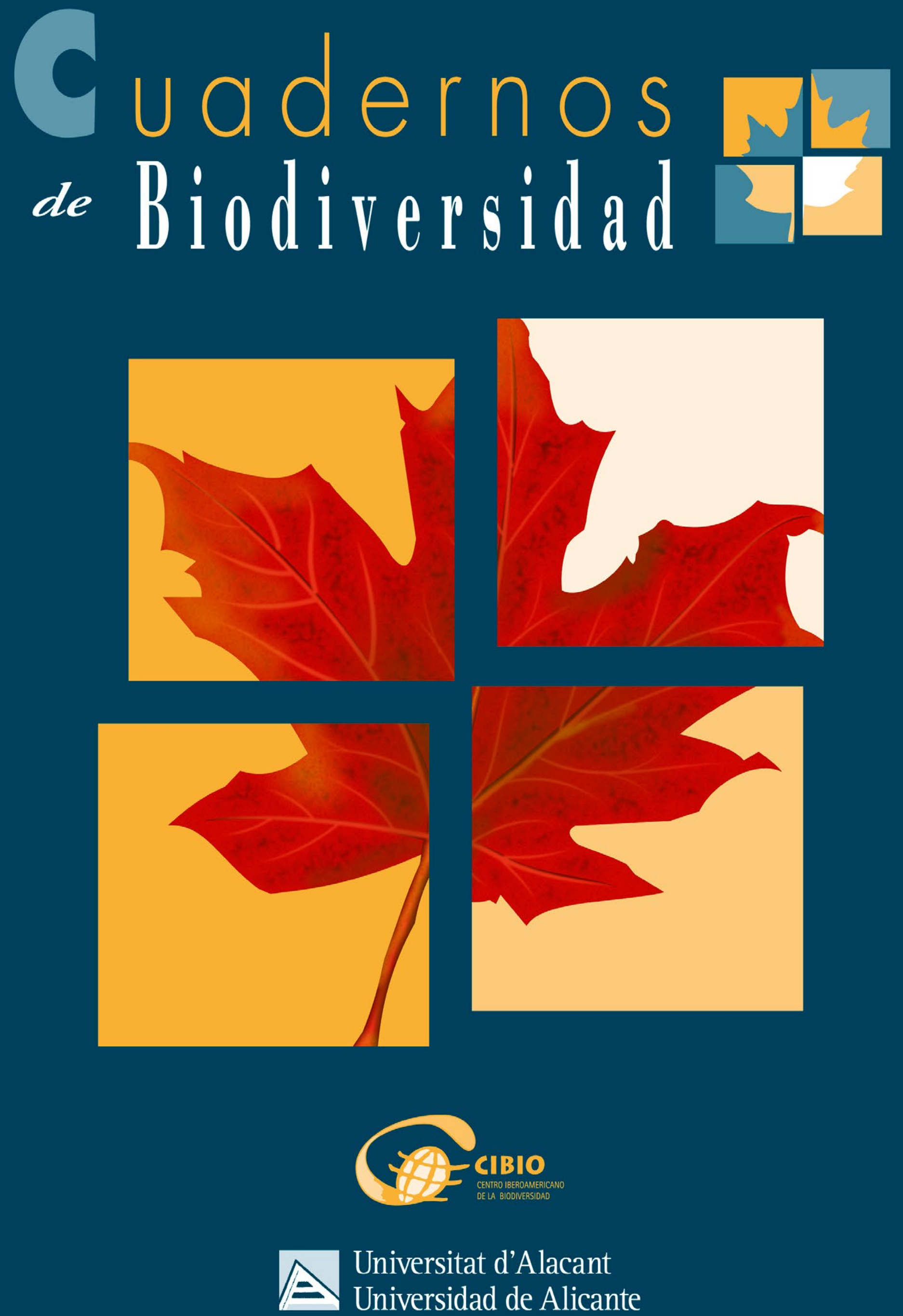


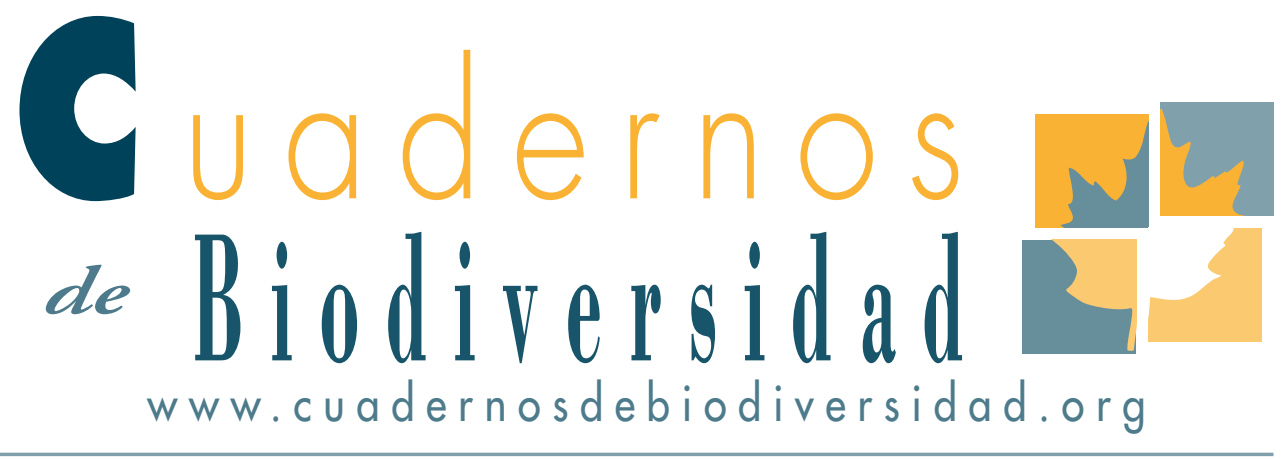

\section{Avances en la taxonomía de los Anomalini (Coleoptera: Scarabaeidae: Rutelinae) de Costa Rica}

\section{Advances in the taxonomy of Costa Rican Anomalini}

\section{Filippini ${ }^{1}$}

i Avda San Pedro del Valle i 895, 4I 30866 Concepción, Chile

VALENTINA.FILIPPINI@GMAIL.COM

\section{RESUMEN}

Se dan a conocer los principales resultados de los recientes estudios sobre la taxonomía y biodiversidad de los Anomalini de Costa Rica. Se discute además el problema actual del "impedimento taxonómico" y la relevancia de la Taxonomía como disciplina biológica.

Pallabras clave: Anomalini, Costa Rica, taxonomía, impedimento taxonómico

\section{ABSTRACT}

The main results of the recent studies on the taxonomy and biodiversity of the Anomalini of Costa Rica are presented. Additionally, the current problem of taxonomic impediment and the relevance of taxonomy as a biological discipline are discussed.

Key words: Anomalini, Costa Rica, Taxonomy, taxonomic impediment 


\section{INTRODUCCIÓN}

Los puntos calientes de biodiversidad son un escenario privilegiado para los diferentes campos de la investigación biológica: la alta biodiversidad, que conlleva ricas redes de interacciones entre especies, las hacen ideales para estudios de ecología, evolución y biodiversidad. Sin embargo, la misma riqueza de especies hace que muchas veces la biodiversidad real de estos lugares no sea bien conocida, sobre todo en grupo de animales pequeńos y numerosos como los insectos.

Esta situación se presentó durante los estudios del grupo de investigación Biodiversidad y Biotecnología aplicadas a la Conservación del Instituto
CIBIO (Universidad de Alicante) en el marco de diferentes proyectos de conservación y de estudio de bioindicadores llevados a cabo en Costa Rica (AECID. A/023060/09, A/4426/05 y A/6788/06) (Figuras 1-2). El acceso a la rica colección del Instituto Nacional de Biodiversidad de Costa Rica se reveló fundamental para la identificación de varios grupos de escarabeidos utilizados en nuestra investigación, sin embargo, para la subtribu Anomalini (Scarabaeidae: Rutelini), la mayoría de las especies estaba sin identificar.

Por este motivo, parte de nuestra investigación, se enfocó en aclarar la taxonomía de los Anomalini, en especial los géneros Anomala y Callistethus, que presentan una elevada diversidad y son poco estudiados.

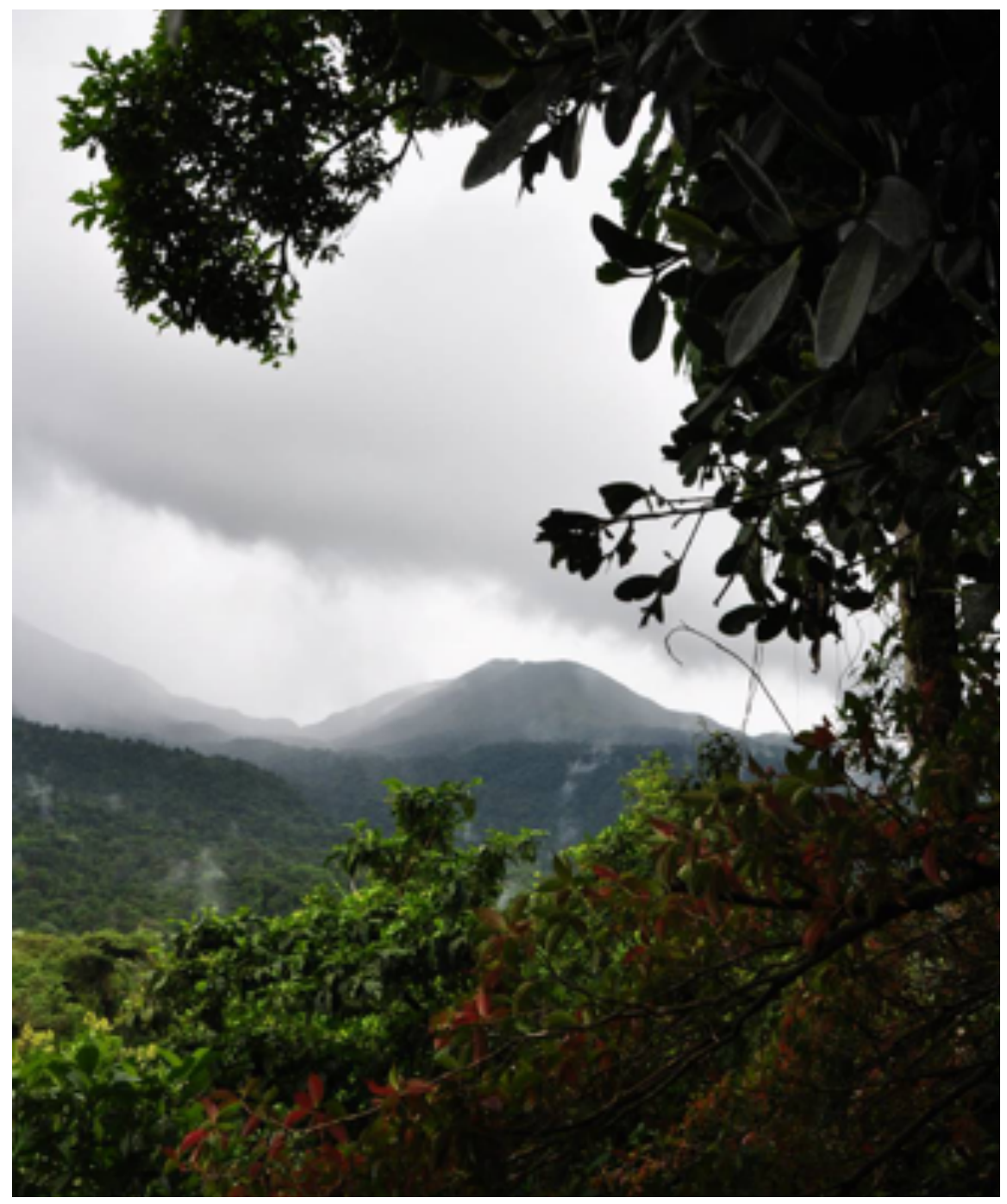

Figura 1. El Parque Nacional Volcán Tenorio, Costa Rica, donde se desarrollaron parte de las investigaciones. Foto de V. Filippini 


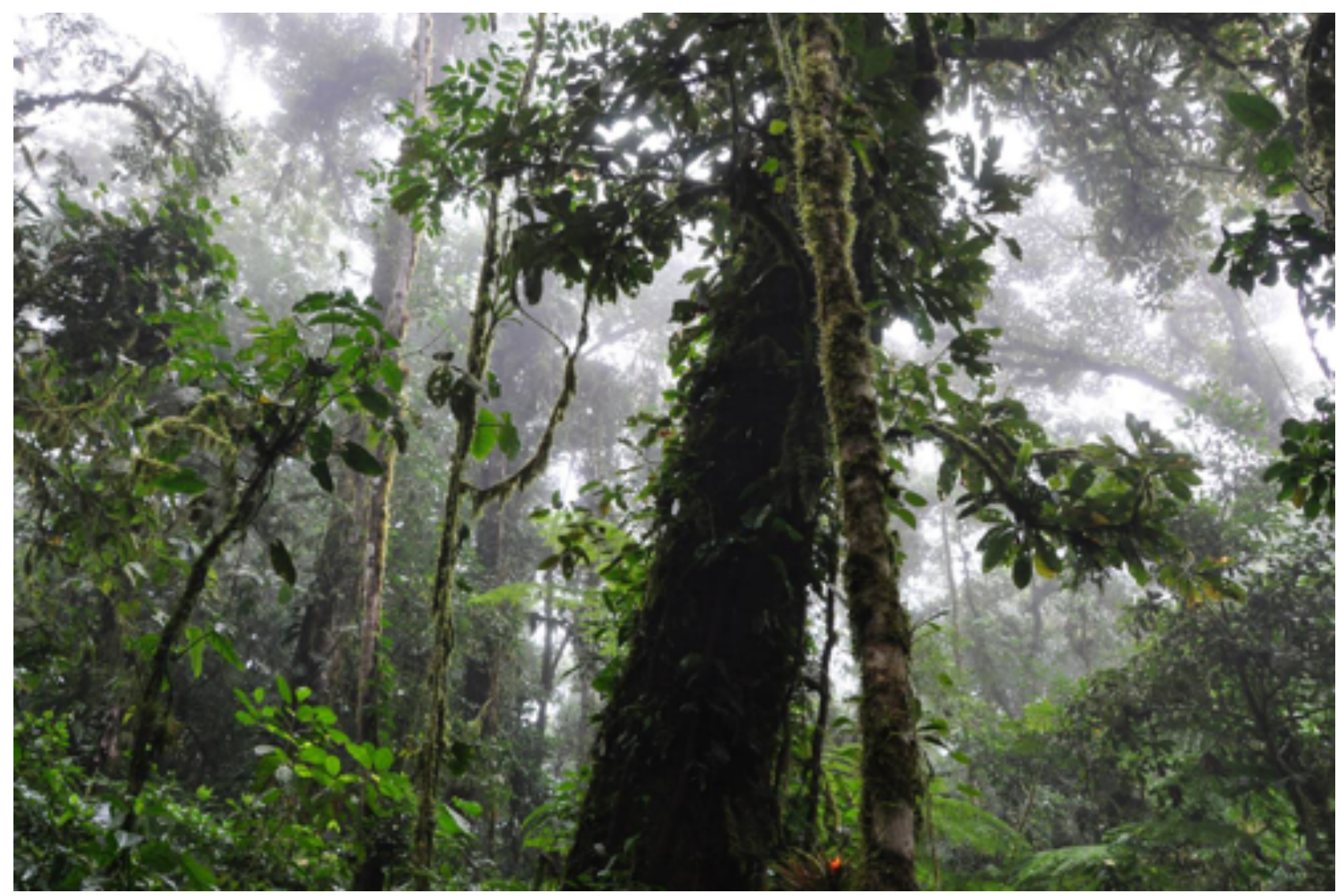

Figura 2. Bosque de niebla, uno de los ambientes donde se encuentran Anomalinos (S. Ramón, Alajuela, Costa Rica). Foto de V. Filippini

\section{LOS ANOMALINI DE COSTA RICA}

La principal dificultad en la identificación de los anomalinos residía en el hecho de que la única manera para una identificación confiable era la comparación con el material tipo. Esto se debe a la antigüedad de las descripciones de la mayoría de las especies (finales del siglo XIX- inicio XX), muy cortas respecto a los estándares actuales, y con insuficientes ilustraciones, y la falta de publicaciones como claves de identificación o revisiones.

El trabajo taxonómico se desarrolló con el estudio morfológico de las morfoespecies conservadas en las colecciones del CIBIO y del INBio, y su comparación con material tipo de las especies neotropicales (en mayoría conservadas en los museos de historia natural de Berlín, Londres y París).

La consulta de material tipo en diferentes museos permitió no solamente comprobar que la mayoría de las morfoespecies eran nuevas especies para la ciencia, sino también permitió identificar 14 especies adicionales entre las morfoespecies, y detectar 14 identificaciones previas erróneas (la especie identificada con un cierto nombre no correspondía al tipo).

Se describieron un total de 58 especies nuevas de Anomalay Callistethus (Figuras 3-17), que junto a las nuevas identificaciones y las correcciones elevaron el número de especies conocidas de Anomalini en Costa Rica de 64 a 120 (Filippini et al., 2013, 2014, 2015a-e, 2016).

Esto hace de Costa Rica el país con más diversidad de Anomalini en América Latina. Más que la biodiversidad real, este dato enfatiza la falta de inventarios faunísticos y estudios taxonómicos en otros países; por ejemplo, en Panamá se registraron sólo 42 especies de Anomalini, (Ratcliffe, 2002), y sólo 79 en Ecuador que tiene una superficie cinco veces mayor a la de Costa Rica (Paucar- Cabrera, 2005). 

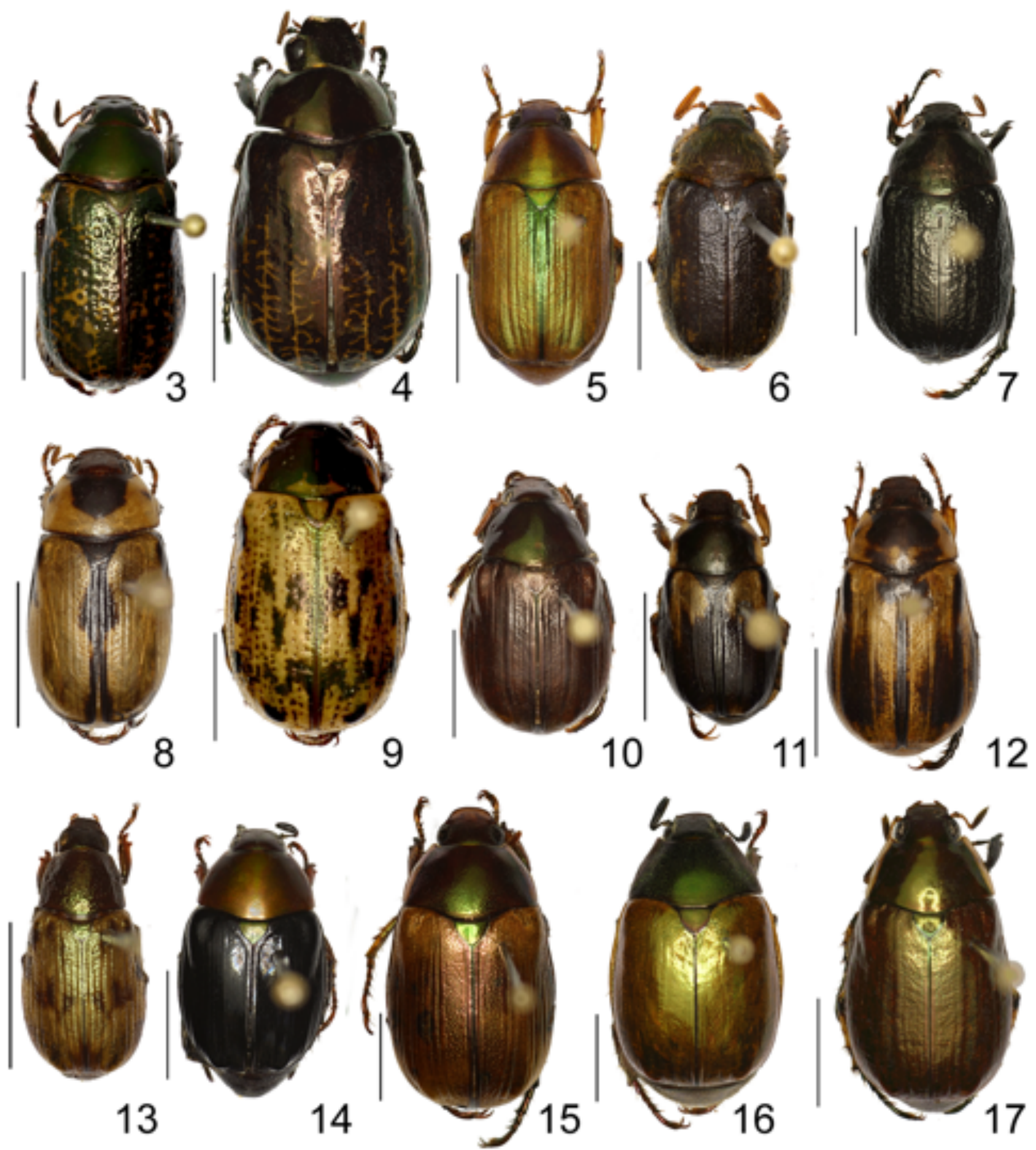

Figuras 3-17. Algunas de las especies descritas durante la investigación: 3. Anomala aereiventris. 4. A. aglaos. 5. A. arthuri. 6. A. atrivillosa. 7. A. cupreovariolosa. 8. A. cyclops. 9. A. eusticta. 10. A. ferrea. 11. A. mersa. 12. A. pincelada. 13. A. strigodermoides. 14. Callistethus carbo. 15. C. lativittis. 16. C. stannibractea. 17. C. yalizo. 
Se puede decir con seguridad que estos números no reflejan la verdadera diversidad de estos países, y que la mayor parte de la diversidad de Anomalini en el Neotrópico esté todavía por descubrir.

Hace falta destacar que este trabajo no hubiera sido posible sin las extensas campañas de recolección de ejemplares, que a lo largo de 20 años fueron a formar la colección del INBio, de la cual proviene la mayoría del material para las descripciones de nuevas especies. Estas deben basarse sobre un espécimen físico, y la recolecta del ejemplar es sólo el primer paso. Muchas de las especies de Anomalinos y otros grupos estaban determinadas, gracias al esfuerzo del personal del Instituto y de colaboradores externos. Sin embargo las especies indeterminadas podrían haberse quedado como tales por décadas, y esto pasa en la mayoría de las colecciones. Esto se debe a que la identificación de una especie, sobre todo de organismos pequeños como los insectos, y de regiones tan ricas como los trópicos, no es sencilla. En definitiva necesita de un experto del grupo y de publicaciones con claves para la identificación de las especies si es que las hay.

Esta dificultad en acceder al conocimiento taxonómico, o la falta de ello, se conoce como impedimento taxonómico

\section{EL IMPEDIMENTO TAXONÓMICO}

Hace más de 20 años que el mundo académico es consciente de la brecha entre la urgencia para conocer la biodiversidad del planeta (con un 90\% estimado de especies todavía desconocidas [Wägele et al., 2011]) y la escasez de taxónomos para identificar y describir estas especies. La Convention on Biological Diversity realizada en 1995 bautizó este problema como "taxonomic impediment" (Evenhuis, 2007).

Entre las causas reconocidas de la escasez de taxónomos está la dificultad para obtener financiación para investigaciones de taxonomía, la resistencia que encuentran los taxónomos a la hora de publicar sus investigaciones en revistas de impacto, la escasa estima de sus estudios por parte de los colegas, que ven en la taxonomía un trabajo más bien burocrático, sin desafíos intelectuales (Wägele et al., 2011).
El reconocimiento del problema ha llevado al desarrollo de varios programas de formación de taxónomos, por ejemplo Global Taxonomy Initiative (https://www.cbd.int/gti/default.shtml) y Partnerships for Enhancing Expertise in Taxonomy (PEET) (U.S. National Science Foundation).

No obstante, aún quien recibe o tiene formación en taxonomía (por ejemplo los becarios del proyecto PEET) termina por trabajar en otras disciplinas, por la dificultad en encontrar trabajo como taxónomo (Agnarsson y Kuntner, 2007).

Es revelador a este propósito saber que en Europa, más del $60 \%$ de los nuevos taxones está descrito por taxónomos aficionados (Fontaine et al., 2012).

Las soluciones sugeridas son varias, como incrementar la formación de nuevos taxónomos, incrementar los fondos económicos destinados a revisiones y estudios taxonómicos, citar siempre al autor de una especie aún en trabajos no taxonómicos, citar a los trabajos utilizados para identificar a las especies objeto de un estudio en el apartado de materiales y métodos, medir la importancia de los trabajos taxonómicos de una manera no basada sobre el número de citas (Impact Factor), convertir la taxonomía en una ciencia más "moderna" implementando por ejemplo el bar-coding en vez de una taxonomía tradicional, o facilitar el intercambio de información taxonómica a través de internet (por ejemplo mediante la digitalización de las colecciones: https://www.idigbio.org/, http://www.zoosphere. net/, http://www.gbif.org/) (Ebach et al., 2011; Wägele et al., 2011).

La discusión sobre estas o nuevas medidas sigue, y aunque cada año se describan cerca de 17000 especies (Fontaine et al., 2012), muchas más faltan por descubrir, y el problema del impedimento taxonómico sigue vigente. El primer paso es un cambio en la concepción del mundo científico hacia la taxonomía. La taxonomía es la base para las otras disciplinas biológicas (Wägele et al. 2011); y debe verse como "big science" (Wheeler et al., 2004).

En la "crisis de biodiversidad" que estamos viviendo, en la cual se extinguen más especies de las que se descubren, no maravilla el hecho de que una encuesta hecha entre el público general del sur-oeste 
de Estados Unidos (Ebach et al. 2011), la mayoría de las personas consideraran muy importante que los científicos se dedicaran a explorar y documentar nuevas especies, y que la taxonomía es una ciencia relevante.

¿Porque entre los mismos científicos no es así? Una de las principales metas de la biología es saber que especies viven en nuestro planeta (Agnarsson y Kuntner, 2007).

Lejos de ser una disciplina aburrida y repetitiva, la taxonomía tiene la fascinación de la poética definición de E.O. Wilson (2004): una "exploración pionera de la vida en un planeta poco conocido". Para descubrir, o redescrubrir, como disfrutar de la taxonomía, Evenhuis (2007) señala 8 pasos para alcanzar la "iluminación y el nirvana taxonómico", que sin duda, aún si la iluminación no llega, ayudan a alcanzar una alta calidad y utilidad en este trabajo.

\section{REFERENCIAS}

Agnarsson, I. \& Kuntner, M. (2007). Taxonomy in a Changing World: Seeking Solutions for a Science in Crisis. Systematic Biology, 56(3): 531-539. https://doi. org/10.1080/10635150701424546

Ebach, M. C., Valdecasas, A. G. \& Wheeler, Q. D. (2011). Impediments to taxonomy and users of tax-onomy: Accessibility and impact evaluation. Cladistics, 27(5): 550-557. https://doi.org/10.1111/j.1096-0031.2011.00348.x

Evenhuis, N. L. (2007). Helping solve the 'other' taxonomic impediment: Completing the Eight Steps to Total Enlightenment and Taxonomic Nirvana. Zootaxa, 12(1494): 3-12.

Filippini, V., Micó, E. \& Galante, E. (2013). Redescription of Anomala eucoma Bates, 1888 and a de-scription of three new species from Costa Rica (Coleoptera : Scarabaeidae : Rutelinae). Zootaxa, 3670(2): 255-273. https://doi. org/10.11646/zootaxa.3670.2.9

Filippini, V., Micó, E. \& Galante, E. (2014). Description of eight new Anomala species from Costa Rica (Coleoptera: Scarabaeidae: Rutelinae). Journal of Entomology and Zoology Studies, 2(6): 107-122.

Filippini, V., Galante, E. \& Micó, E. (2015a). Descriptions of New Species of Anomala Samouelle ( Col-eoptera : Scarabaeidae : Rutelinae ) from Costa Rica. The Coleopterists Bulletin, 69(3): 463-476. https://doi. org/10.1649/0010-065X-69.3.463
Filippini, V., Galante, E. \& Micó, E. (2015b). Description of six new species of Anomalini from Costa Rica (Coleoptera: Scarabaeidae: Rutelinae). Zootaxa, 3948(1): 24-40. https://doi.org/10.11646/zootaxa.3948.1.2

Filippini, V., Galante, E. \& Micó, E. (2015c). The genus Callistethus (Coleoptera: Scarabaeidae: Rute-linae) in the Neotropics: new data and new species from Costa Rica. Arthropod Systematics \& Phylogeny, 73(2): 199-238.

Filippini, V., Micó, E. \& Galante, E. (2015d). Anomala trapezifera species-group: a burst of diversity (Coleoptera: Scarabaeidae: Rutelinae). Annales de La Société Entomologique de France (N.S.): International Journal of Entomology, 51(2): 93-139. https://doi.org/10.1080/00 379271.2015 .1054646

Filippini, V., Micó, E. \& Galante, E. (2015e). Description of two cryptic species of Anomala Samouelle (Coleoptera : Scarabaeidae : Rutelinae ) from Costa Rica. Dugesiana, 22(2): 215-220.

Filippini, V., Micó, E. \& Galante, E. (2016). Checklist and identification key of Anomalini (Coleoptera, Scarabaeidae, Rutelinae) of Costa Rica. ZooKeys, 621: 63-136. https://doi.org/10.3897/zookeys.621.7565

Fontaine, B., van Achterberg, K., Alonso-Zarazaga, M. A., Araujo, R., Asche, M., Aspöck, H., Aspöck, [...] \& Bouchet, P. (2012). New species in the old world: Europe as a frontier in biodiversity exploration, a Test Bed for 21st century taxonomy. PLoS ONE, 7(5): e36881. https:// doi.org/10.1371/journal.pone.0036881

Ratcliffe, B. C. (2002). A checklist of the Scarabaeoidea (Coleoptera) of Panama. Zootaxa, 32: 1-48.

Paucar-Cabrera, A. (2005). A catalog and distributional analysis of the Rutelinae (Coleoptera: Scarabaeidae) of Ecuador. Zootaxa, 948: 1-92.

Wägele, H., Klussmann-Kolb, A., Kuhlmann, M., Haszprunar, G., Lindberg, D., Koch, A. \& Wägele, J. W. (2011). The taxonomist - an endangered race. A practical proposal for its survival. Frontiers in Zoology, 8(1): 25. https://doi.org/10.1186/1742-9994-8-25

Wheeler, Q. D. (2004). Taxonomy: Impediment or Expedient? Science, 303(5656): 285-285. https://doi. org/10.1126/science.303.5656.285

Wilson, E. O. (2004). Taxonomy as a fundamental discipline. Philosophical Transactions of the Royal Society of London. Series B, Biological Sciences, 359(1444): 739. https://doi. org/10.1098/rstb.2003.1440 